kleine Exzentrizitäten. Außerdem tritt, bei kleinen Krümmungen gut sichtbar, eine Parallelverschiebung zwischen Meß- und Rechenkurven auf, die jedoch innerhalb der Fehlergrenze der Messungen liegt.

Exakte Übereinstimmung im Verlauf findet man daher erwartungsgemäß für kleine Krümmungen. Die beste Übereinstimmung der Absolutwerte ist bei ca. 4 A festzustellen, dort liegen die Differenzen zwischen $12 \%$ und $3 \%$.

\section{Zusammenfassung}

Die exzentrische Lage des gekrümmten, wandstabilisierten Bogens wird hervorgerufen durch das Zusammenwirken dreier Unsymmetrien: der des elektrischen Feldes, der der Wärmeleitung und der des Eigenmagnetfeldes. Den größten Anteil an der Verlagerung des Bogens nach innen hat die Unsymmetrie der elektrischen Feldstärke. In der gleichen
Richtung, aber schwächer, wirkt die Wärmeleitungsunsymmetrie. Die Verschiebung nach innen infolge dieser zwei Unsymmetrien wird teilweise durch die eigenmagnetische Strömung kompensiert, die den Bogen etwas nach außen mitnimmt. Alle drei Effekte nchmen mit der Krümmung zu. Unmittelbar stromabhängig ist nur der dritte; die zwei ersten werden es mittelbar, wenn sich mit dem Strom gemäß der Charakteristik die elektrische Feldstärke und damit die Leitfähigkeit ändert. Wegen des thermischen Nichtgleichgewichts beim Argonniederstrombogen wird die Leitfähigkeit von der Feldstärke und damit vom Strom abhängig. Daher ist hier das Nichtgleichgewicht Ursache für die starke Zunahme der Exzentrizitäten mit abnehmendem Strom.

Der Verfasser dankt Herrn Prof. Dr. H. Maecker herzlich für die Themenstellung und Förderung der Arbeit und der Deutschen Forschungsgemeinschaft für die Gewährung einer Sachbeihilfe.

\title{
Quantitative Ausmessung des Brom-Affinitätskontinuums und Bestimmung der Detachment- und Attachment-Querschnitte
}

\author{
H. Frank, M. Neiger und H.-P. Popp * \\ Elektrophysikalisches Institut der Technischen Universität München \\ (Z. Naturforsch. 25 a, 1617-1626 [1970] ; eingegangen am 17. September 1970)
}

\begin{abstract}
A wall stabilized low-current cylindric arc was used to produce the radiation of the negative Bromine-ions. The radiation consists of an affinity-continuum with a long-wave threshold of $3682 \AA$, yielding an electron affinity for Bromine of $3.366 \mathrm{eV}$, and of an intense electron-atom Bremsstrahlung in the visible. Intensity measurements of the continua allow the determination of the photodetachment- and attachment-cross-sections of Bromine and also the determination of the mean elastic cross-section of electrons against Bromine atoms.
\end{abstract}

Die Absolutmessung der kontinuierlichen Strahlung, wie sie bei der Anlagerung freier Elektronen an neutrale Atome auftritt, erlaubt zusammen mit einer Plasmatemperaturmessung die Bestimmung des Detachment- und Attachment-Querschnittes. Zur Erzeugung des Affinitätskontinuums und zu dessen quantitativer Messung eignet sich besonders der zylindersymmetrische, wandstabilisierte Niederstromlichtbogen ${ }^{1-3}$. Es sind jetzt aber auch quantitative Messungen mit Hilfe des Stoßwellenrohres von ROTHE ${ }^{4}$ bekanntgeworden.

* OSRAM-Studiengesellschaft München.

Sonderdruckanforderungen an Prof. Dr. H. Maecker, Elektrophysikalisches Institut der Technischen Universität München, D-8000 München 2, Arcisstraße 21 .

1 H.-P. Popp, Z. Naturforsch. 20 a, 642 [1965].
Für grundlegende Untersuchungen der Strahlung negativer Ionen eignen sich die Halogene wegen ihrer großen Elektronenaffinität ${ }^{5}(3,0$ bis $3,6 \mathrm{eV})$ und dem sich daraus ergebenden günstigen Spektralbereich besonders gut. So wurden bisher die Detachment-Querschnitte der Halogene Fluor ${ }^{2}$ und Chlor ${ }^{3}$ aus Messungen am Lichtbogen und der Halogene Chlor, Brom, Jod ${ }^{4}$ aus Messungen am Stoßwellenrohr bestimmt. Die ermittelten Detachment-Querschnitte, wie sie im Lichtbogen ${ }^{3}$ und im Stoßwellenrohr ${ }^{4}$ gewonnen wurden, stimmen weitgehend mit-

2 H.-P. Popp, Z. Naturforsch. 22 a, 254 [1967].

3 G. Mück u. H.-P. Popp, Z. Naturforsch. 23 a, 1213 [1968].

4 D. E. Rothe, Phys. Rev. 177, 93 [1969].

5 H.-P. Popp, 8. Intern. Conf. Phenomena Ionized Gases, Springer-Verlag, Wien 1967, S. 448. 
einander und mit der Theorie von RoBinson und Geltman $^{6}$ überein. Die Übereinstimmung ergibt sich auch für die hier berichteten Messungen am Brom-Niederstrombogen.

Neben dem Brom-Affinitätskontinuum wurde im Brom-Niederstrombogen ein starker kontinuierlicher Untergrund beobachtet, der mit der Kramers-Unsöldschen Theorie ${ }^{7,8}$ auch in der verbesserten Form ${ }^{9,10}$ nicht als Rekombinations- und frei-freiKontinuum der Elektronen und positiven Ionen erklärt werden kann. Mit der Theorie von Kas'yanov und Starostin ${ }^{11}$, welche den Zusammenhang zwischen dem differentiellen inelastischen Streuquerschnitt und dem totalen elastischen Streuquerschnitt für Elektron-Atom-Streuung angibt, läßt sich eine Beziehung zwischen dem spektralen Emissionskoeffizienten der frei-frei-Minus-Strahlung (Elektronenbremsstrahlung im Feld der Atome) und einem mittleren totalen elastischen Streuquerschnitt finden. Die beobachtete starke Strahlung des Untergrundes wird unter Annahme eines mittleren totalen elastischen Streuquerschnitts für Elektronen gegen Bromatome von $1,6 \cdot 10^{-14} \mathrm{~cm}^{2}$ als frei-frei-Minus-Strahlung richtig wiedergegeben.

Der Einfluß des thermischen Nichtgleichgewichtes wird experimentell und theoretisch geprüft und Gleichgewicht für die ausgewerteten Messungen gefunden.

\section{Messungen}

\subsection{Der Brom-Lichtbogen}

Um ein Kondensieren des Bromgases im Quarzbogengefä $\beta \mathrm{zu}$ verhindern, mu $\beta$ die Temperatur der kältesten Brom-Bogengefäß-Stelle über $57^{\circ} \mathrm{C}$ liegen. Dieser Temperaturwert ergibt sich aus der Dampfdruckkurve des Broms ${ }^{12}$ für den Druck unter Versuchsbedingungen von 715 Torr. Eine Wasserkühlung des Quarzrohres scheidet aus diesem Grunde bei kleineren Bogenstromstärken aus. Nach ersten Kühlversuchen mit über $60^{\circ} \mathrm{C}$ heißem Wärmeübertragungsöl wird wegen der dabei auf ca. $60 \mathrm{~W} / \mathrm{cm}$ Bogenlänge begrenzten Wandbelastung das Lichtbogengefäß mit radial zuströmender Preßluft vor der Zerstörung bewahrt. Die Belastungsgrenze des Quarzbogengefäßes von $8 \mathrm{~mm}$ Innendurchmesser betrug bei der zur Verfügung stehenden Preßluftmenge ca. $150 \mathrm{~W} / \mathrm{cm}$.

Um die Strahlungsabsorption kalter Bromschichten des end-on beobachteten Bromlichtbogens und eine Zer-

${ }^{6}$ E. J. Robinson u. S. Geltman, Phys. Rev. 153, 153 [1967].

7 H. A. Kramers, Phil. Mag. 46, 836 [1923].

8 A. Unsöld, Ann. Phys. 33, 607 [1938].

9 H. Maecker u. Th. Peters, Z. Phys. 139, 448 [1954]. störung der Elektroden durch das Halogen sowie ein Beschlagen der kalten Beobachtungsfenster mit Brom zu vermeiden, werden die Lichtbogenenden mit Argon gespült. In Abb. 1 ist das verwendete Lichtbogengefäß skizziert. Die Elektroden sind seitlich angeordnet, die kurzen, vor der Bromlichtbogensäule liegenden Argonplasmaschichten besitzen gegenüber dem Brom eine so geringe Strahldichte, daß ihr Emissionsbeitrag vernachlässigt werden kann (im end-on-Spektrum sind keine Argonlinien sichtbar). Die Länge der Bromlichtbogensäule wird deshalb von den Gasaustrittsöffnungen bestimmt und beträgt $10 \mathrm{~cm}$. Die eingeströmte Argongasmenge liegt bei jeweils $4 \mathrm{~cm}^{3} / \mathrm{s}$.

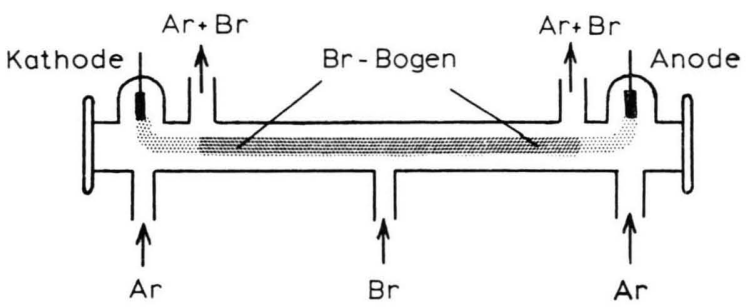

Abb. 1. Bromlichtbogengefäß.

Das dem Lichtbogengefäß über eine beheizte Leitung und eine Mehrfachkapillare zugeführte Bromgas wird in einem Thermostaten mit kontinuierlicher elektronischer Regelung durch Verdampfen erzeugt. Der Überdruck im Verdampfungsgefäß gegenüber dem Bromlichtbogen beträgt ca. 50 Torr, die Arbeitstemperatur des Wärmebades liegt somit bei $59,6{ }^{\circ} \mathrm{C}$. Um Schwankungen der durch die Kapillare auf ca. $4 \mathrm{~cm}^{3} / \mathrm{s}$ begrenzten Bromgasströmung kleiner als $5 \%$ zu halten, muß das Wärmebad des Verdampfungsgefäßes besser als $0,1{ }^{\circ} \mathrm{C}$ geregelt sein. Die Zündung des Lichtbogens erfolgt in Argon bei niederem Druck durch Hochspannungsdurchschlag.

\subsection{Registrierung der Spektren}

Der Kern des Brom-Lichtbogens wird end-on mit kleinem Öffnungswinkel $(1: 250)$ auf den $1 \mathrm{~mm}$ hohen Spalt eines Spektrographen (Steinheil Universal mit zwei Quarzprismen) abgebildet und das Spektrum mit einem Photomultiplier-Zusatz photoelektrisch registriert. Zur Absoluteichung wurde als Strahlungsnormal der Anodenbrennfleck des Graphit-Niederstrombogens nach EULER ${ }^{13}$ verwendet. Die Stabilität der Bogenentladung wurde über lange Zeiten ( $>1$ Std.) durch Registrierung des Spektrums bei festgehaltener Wellenlänge überprüft und maximale Abweichungen von nur $2 \%$ gefunden.

Die spektrale Registrierung des Lichtbogenkerns zeigt im ultravioletten Spektralbereich das von Linien weitgehend ungestörte Brom-Affinitätskontinuum in

10 M. Bibermann u. G. E. Normann, Optics and Spectr. 8, 230 [1966].

11 V. Kas'yanov u. A. Starostin, Sov. Phys. JETP 21, 193 [1965].

12 W. Ramsay u. S. Joung, J. Chem. Soc. 49, 453 [1886]. 
einem Intensitätsverhältnis von ca. $3: 1$ zu dem im Sichtbaren sehr starken Untergrund.

Zur Bestimmung der Achstemperaturen bei verschiedenen Bogenstromstärken werden Absolutmessungen der Linienintensität der Bromlinie 4981,15 $\AA$ ausgeführt.

\subsection{Charakteristikmessungen}

Die Abschätzung des lokalen thermischen Gleichgewichtes erfordert unter anderem die Kenntnis der Feldstärke-Stromstärke-Charakteristik der Brombogensäule. Eine direkte Messung der Feldstärke durch Sonden ist auch in der Argon-Brom-Mischzone bei den Gasauslässen nicht möglich, da das Halogen die Wolframsonden chemisch zerstört. Die Feldstärke der Bromentladung wurde deshalb indirekt durch Messung der Gesamtbogenspannung bei Bromzufuhr und Messung der Spannungen zwischen den Elektroden und Sonden in den Gasauslässen bei reinem Argongas bestimmt. Die Subtraktion der so bestimmten Elektrodenfälle und ArgonTeilbogenspannungen von der Gesamtspannung bei Bromzufuhr ergibt mit ausreichender Genauigkeit $( \pm 7 \%)$ die Spannung des Brom-Teilbogens. Die nach Division durch die Brombogenlänge $(10 \mathrm{~cm})$ erhaltene Feldstärke des Bromlichtbogens ist in Abb. 2 als Funktion des Bogenstroms dargestellt. Die Feldstärken liegen nur wenig über denen des reinen Argonbogens, die zusammen mit der im Brombogen umgesetzten Leistung in Abb. 2 eingetragen sind.

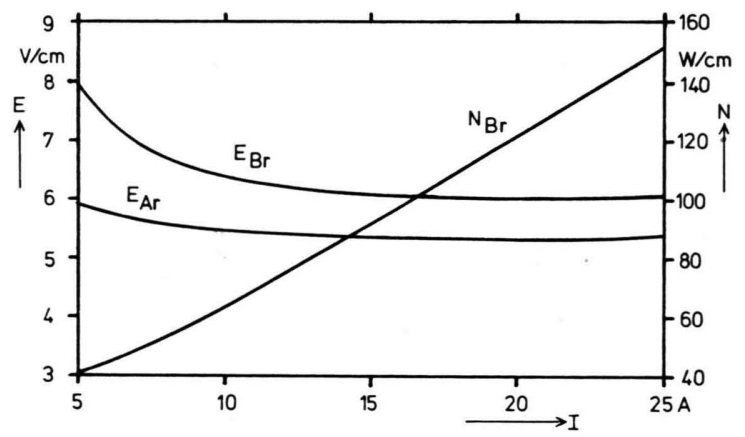

Abb. 2. Feldstärke-Stromstärke-Charakteristik für den Bromund Argonlichtbogen in einem Bogengefäß von $8 \mathrm{~mm}$ Innendurchmesser bei Normaldruck sowie die im Bromlichtbogen umgesetzte elektrische Leistung.

\section{Auswertung}

\subsection{Die Elektronenaffinität von Brom}

Das Brom-Affinitätskontinuum tritt im Bromlichtbogen mit zwei ausgeprägten langwelligen Kanten entsprechend der Anlagerung von freien Elek-

13 J. Euler, Ann. Phys. 6, 11 [1953].

14 R. S. Berry, C. W. Reimann u. G. N. Spokes, J. Chem. Phys. 37, 2278 [1962]. tronen an die beiden Brom-Grundniveaus ${ }^{2} \mathrm{P}_{3 / 2}^{0}$ und ${ }^{2} \mathrm{P}^{0}{ }_{1 / 2}$ auf. Die Kanten zeigen jedoch eine stromstärke- bzw. temperaturabhängige Verschmierung. Zur genauen Bestimmung der Elektronenaffinität aus den Kanten wird deshalb eine von BERRY ${ }^{14}$ vorgeschlagene Methode verwendet. Man legt dazu im kantennahen Wendepunkt, der durch die Verschmierung entsteht, eine Tangente an die Meßkurve und fällt ebenfalls vom Wendepunkt aus das Lot auf den extrapolierten Untergrund. Als Kanteneinsatz wird die Mitte zwischen dem Schnittpunkt der Tangente und dem des Lotes mit dem extrapolierten Untergrund definiert. Damit ergeben sich für die Grenzwellenlängen und für die entsprechenden Energien die Werte:

1. Kante: $3682 \pm 2 \AA$ oder $3,366 \pm 0,003 \mathrm{eV}$

2. Kante: $3240 \pm 2 \AA$ oder $3,825 \pm 0,004 \mathrm{eV}$

Eine Verschiebung der Kanten nach längeren Wellenlängen hin, was gleichbedeutend mit einer Erniedrigung der Elektronenaffinität ist, wird mit zunehmender Stromstärke bzw. Temperatur in Übereinstimmung mit der Theorie ${ }^{15}$ nicht festgestellt. Die Energiedifferenz zwischen den beiden Kanten von $0,459 \mathrm{eV}$ entsprechend $3703 \mathrm{~cm}^{-1}$ stimmt innerhalb der Meßgenauigkeit von $\pm 50 \mathrm{~cm}^{-1}$ mit der Energiedifferenz der beiden Grundzustände des Bromatoms nach MOORE ${ }^{16}$ von $3685 \mathrm{~cm}^{-1}$ überein.

Die Elektronenaffinität des Brom ergibt sich deshalb aus der ersten Kante zu

$$
\mathrm{EA}(\mathrm{Br})=3,366 \pm 0,003 \mathrm{eV} \text {. }
$$

Messungen der langwelligen Kanten des Bromaffinitätskontinuums von BERRY ${ }^{14,17}$ mit Hilfe des Stoßwellenrohres ergaben für die Elektronenaffinität Werte von $3,358 \mathrm{eV} \pm 0,005 \mathrm{eV}$ in Absorption und $3,362 \mathrm{eV} \pm 0,004 \mathrm{eV}$ in Emission. Innerhalb der Fehlergrenzen stimmen diese Werte mit den von uns gemessenen überein.

\subsection{Plasmazusammensetzung}

Die Plasmazusammensetzung wird zur Temperaturbestimmung aus der Absolutintensität einer Bromlinie und zur Berechnung mikroskopischer Größen (atomarer Konstanten) aus den makroskopischen Meßgrößen benötigt. Da der Lichtbogen in reinem

15 H. R. Griem, Plasma Spectroscopy, McGraw-Hill, New York 1964, S. 139.

16 C. E. Moore, Atomic Energy Levels, Vol. 2, NBS Circulaire 467. 
Brom betrieben wird, reichen zur Berechnung der Dichten von Brommolekülen, Atomen, positiven und negativen Ionen sowie Elektronen die folgenden Gleichungen aus :

1. Massenwirkungsgesetz für die Dissoziationsreaktion,

2. Saha-Gleichungen für die Ionisationsreaktion und die Elektronenanlagerungsreaktion,

3. Daltonsches Partialdruckgesetz,

4. Quasineutralitätsbedingung.

Zur exakten Lösung des Gleichungssystems steht ein Rechenprogramm von FRIE $^{18}$ zur Verfügung. Für die Rechnung wurden folgende Werte verwendet: Normaldruck $=715$ Torr, $\quad$ Dissoziationsenergie ${ }^{19}=$ $1,97 \mathrm{eV}$, Ionisationsenergie ${ }^{16}=11,84 \mathrm{eV}$, Elektronenaffinität $=3,366$, Zustandssummen von Atom und Ion nach Drawin und FelenboK ${ }^{20}$, Zustandssumme Minusion $=1$. Die Zustandssummen des Moleküls wurden mit einem Rechenprogramm nach STUPOCHENKO ${ }^{21}$ mit den Molekülkonstanten nach HERZBerg ${ }^{19}$ bestimmt. Die Erniedrigung der Ionisationsenergie wurde nach GRIEM ${ }^{15}$ berücksichtigt.

Das Ergebnis der Teilchendichtenberechnung ist in Abb. 3 in Form eines Teilchendichtediagramms gezeigt.

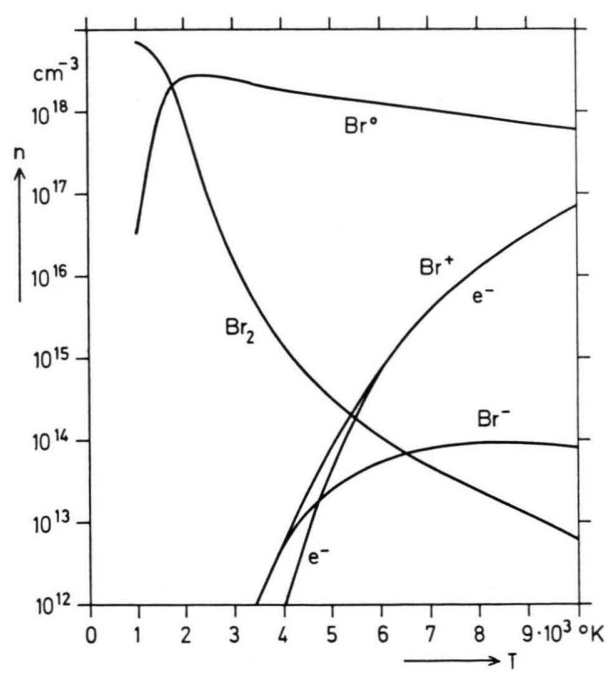

Abb. 3. Teilchendichtediagramm für Bromgas mit 715 Torr Gesamtdruck.

17 R. S. Berry u. C. W. Reimann, J. Chem. Phys. 38, 1540 [1963].

18 W. Frie, Z. Phys. 201, 269 [1967].

19 G. Herzberg, Spectra of Diatomic Molecules, 2. Aufl., 11. Druck, van Nostrand, Princeton 1966.

$20 \mathrm{H}$. W. Drawin u. P. Felenbok, Data for Plasmas in LTE, Gauthier-Villars, Paris 1965.

\subsection{Temperaturbestimmung}

Die Kenntnis der Plasmatemperatur ist für die Anwendung des Teilchendichtediagramms und zur Berechnung des Absorptionskoeffizienten aus den gemessenen Intensitäten mit Hilfe des Kirchhoffschen Gesetzes notwendig.

Die für alle Strahlungsprozesse maßgebende Elektronentemperatur wird aus der Absolutintensität einer geeigneten Br I-Linie ermittelt. In der Literatur sind jedoch weder experimentell gemessene noch theoretisch berechnete Übergangswahrscheinlichkeiten bekanntgeworden, so daß die Übergangswahrscheinlichkeit mit der Coulomb-Approximation nach Bates und DamgaARD ${ }^{22}$ berechnet werden mußte.

Die Br I-Linie 4981,15 $\AA$ erweist sich wegen ihrer isolierten Lage im Spektrum als günstig. Die Klassifikation der beiden zu dieser Linie gehörenden Terme ist die gleiche wie bei der Chlorlinie 4526,2 $\AA$, die in einer früheren Arbeit $^{3}$ zur Temperaturmessung im Chlorplasma verwendet wurde und deren Übergangswahrscheinlichkeit von FOSTER ${ }^{23}$ mit guter Genauigkeit gemessen wurde. Die Berechnung der Übergangswahrscheinlichkeiten für die $\mathrm{Cl}$ I- und Br I-Linien sowie ein Vergleich mit dem experimentellen Wert der Cl I-Linie erlaubt eine Korrektur der Rechnung für die Br I-Linie, die wegen der bei Elementen mit hoher Ordnungszahl zunehmend schwächer werdenden L-S-Kopplung erforderlich ist.

Als Ergebnis der Rechnungen nach BATES und DAMGAARD erhält man:

$$
\begin{array}{ll}
\text { Cl I-Linie 4526,2 } \AA, & A_{n}^{m}=5,5 \cdot 10^{6} \mathrm{~s}^{-1}, \\
\text { Br I-Linie 4981,2 } \AA, & A_{n}^{m}=4,9 \cdot 10^{6} \mathrm{~s}^{-1} .
\end{array}
$$

Der experimentelle Wert von FOSTER ${ }^{23}$ für die Cl ILinie beträgt $A_{n}^{m}=4,1 \cdot 10^{6} \mathrm{~s}^{-1}$.

Wegen der Abweichung von der L-S-Kopplung wird angenommen, daß der Fehler der berechneten Übergangswahrscheinlichkeit der Br I-Linie gleich oder größer ist als bei der Cl I-Linie. Der $A_{n}^{m}$-Wert der Br I-Linie wird deshalb um den gleichen Faktor herabgesetzt, wie er sich aus dem Verhältnis der gemessenen zur gerechneten Übergangswahrscheinlichkeit der Cl I-Linie ergibt. Für die weiteren Rechnun-

21 E. V. Stupochenko et al., in: Physical Gas Dynamics, ed. A. S. Predvoditelev, Pergamon Press, Oxford 1961.

22 D. R. Bates u. A. Damgandd, Phil. Trans. Roy. Soc. Ser. A 242, 101 [1949].

23 E. W. Foster, Proc. Phys. Soc. London 80, 887 [1962]. 
gen wurde deshalb ein $A_{n}^{m}$-Wert für die Br I-Linie $4981,2 \AA$ von

$$
A_{n}^{m}=3,6 \cdot 10^{6} \mathrm{~s}^{-1} \text { (korrigiert) }
$$

verwendet.

Die berechnete Abhängigkeit der Linienintensität der Br I-Linie 4981,2 $\AA$ von der Plasmatemperatur ist in Abb. 4 gezeigt. Neben der oben angegebenen korrigierten Übergangswahrscheinlichkeit wurden tätskontinuum ist einem glatten und relativ starken Untergrund überlagert. Der Verlauf des Affinitätskontinuums nach der zweiten langwelligen Kante ist durch aufsitzende diffuse Molekülbanden bzw. Molekülkontinua gestört. Sie entstehen durch Übergänge aus einem hoch angeregten, gebundenen Molekülzustand in einen ungebundenen Zustand. Die auftretenden Molekülspektren sind in der Literatur ${ }^{24}$ bekannt.

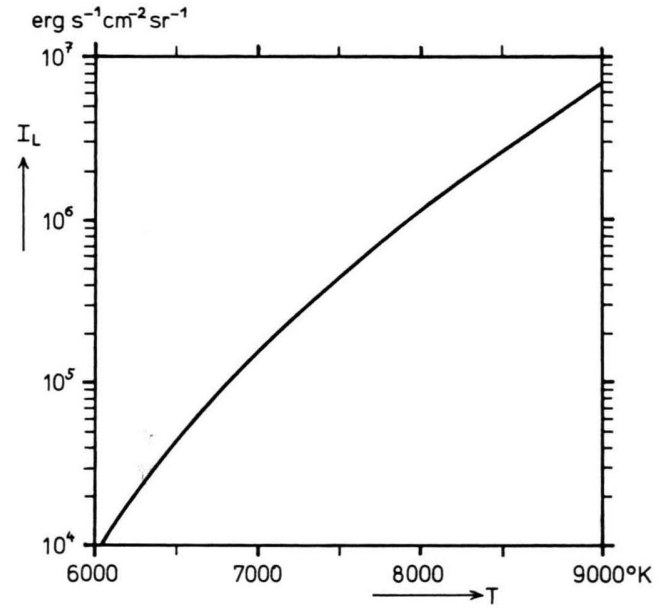

Abb. 4. Linienintensität der Br I-Linie 4981,15 $\AA$ als Funktion der Plasmatemperatur für eine homogene Plasmasäule von $10 \mathrm{~cm}$ Länge und einem Bromgasdruck von 715 Torr.

die folgenden Werte verwendet: Energie des oberen Niveaus ${ }^{16} \quad E_{\mathrm{m}}=87252,57 \mathrm{~cm}^{-1}$, statistisches Gewicht $g_{\mathrm{m}}=4$, Bromzustandssumme $\mathrm{Z}(\mathrm{Br})$ aus ${ }^{20}$, Atomteilchendichte aus Abb. 3, Länge des Lichtbogens $l=10 \mathrm{~cm}$.

Für die bei den Messungen verwendeten Stromstärken von 7, 14,5 und 23,5 Ampere erhält man so Achsentemperaturen von 7150,7600 und $7900{ }^{\circ} \mathrm{K}$. Die Voraussetzung der Emission aus optisch dünner Schicht ist gegeben, da die optische Dicke in Linienmitte $\tau=0,005$ bis 0,03 beträgt.

\subsection{Der Absorptionskoeffizient der kontinuier- lichen Strahlung}

Die im Spektralbereich von 5200 bis $2600 \AA$ von der Bogenachse emittierte gesamte und absolut gemessene Kontinuumsintensität wird mit Hilfe des Kirchhoffschen Gesetzes unter Berücksichtigung der Bogenlänge und der induzierten Emission in den spektralen Verlauf des Absorptionskoeffizienten umgerechnet. In Abb. 5 ist dieser Verlauf für drei verschiedene Temperaturen dargestellt. Das Br-Affini-

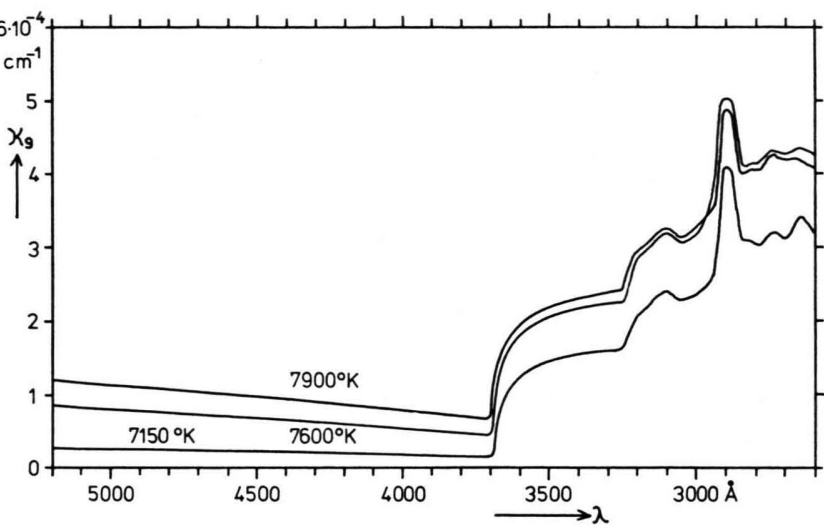

Abb. 5. Spektraler Verlauf des Absorptionskoeffizienten der vom Bromlichtbogen emittierten Kontinua für drei verschiedene Temperaturen im Lichtbogenkern. Rechts das Affinitätskontinuum.

\subsection{Die Trennung des Affinitätskontinuums vom Untergrund und von den Molekülbanden}

Der glatte und zu kurzen Wellenlängen hin fast linear abfallende Untergrund erlaubt es, die Trennung des Affinitätskontinuums wie auch schon im Falle des Fluors ${ }^{2}$ und Chlors ${ }^{3}$ durch eine lineare Extrapolation vorzunehmen. Wie bei der Deutung des Untergrundes in Kapitel 2.7 erklärt wird, ist der Fehler bei der linearen Extrapolationsmethode klein und die Extrapolation theoretisch weitgehend berechtigt.

Weitaus schwieriger ist die Trennung des Affinitätskontinuums von den überlagerten Molekülbanden. Um ein Unterfahren der Banden nach freiem Ermessen zu vermeiden, wird der Verlauf des vom Untergrund getrennten Affinitätskontinuums zusammen mit den Molekülbanden durch die Zahl der negativen Bromteilchen aus dem Teilchendichtediagramm (Abb. 3) geteilt und geprüft, ob sich der so ergebende und noch durch die Molekülbanden ge-

24 K. Asundi u. P. Ventkateswarlu, Ind. J. Phys. 21, 101 [1947]. 
störte atomare Absorptionskoeffizient oder „Detachment-Querschnitt", wie von der Theorie gefordert, temperaturunabhängig verhält. Da nämlich vor dem Affinitätskontinuum keine Linie des negativen Bromions gefunden wurde, d. h. zwischen dem ${ }^{1} \mathrm{~S}_{0}$ Grundzustand des negativen Bromions und den beiden möglichen Ablösungsgrenzen ${ }^{2} \mathrm{P}_{1 / 2}^{0}$ und ${ }^{2} \mathrm{P}_{3 / 2}^{0}$ kein stabiler Zustand existiert, muß der sich ergebende „Detachment-Querschnitt“ temperaturunabhängig werden.

Die Differenz zwischen dem spektralen Verlauf des Absorptionskoeffizienten des Gesamtkontinuums (Abb. 5) und dem gemessenen bzw. extrapolierten Untergrund, geteilt durch die Teilchendichte der negativen Bromionen, ist in Abb. 6 dargestellt. In dem von Banden ungestörten Verlauf des Affinitätskontinuums zwischen erster und zweiter langwelliger Grenze zeigt sich nur für die Temperaturen von 7600 und $7900{ }^{\circ} \mathrm{K}$ eine Übereinstimmung. Der zu der Temperatur von $7150^{\circ} \mathrm{K}$ gehörende Verlauf

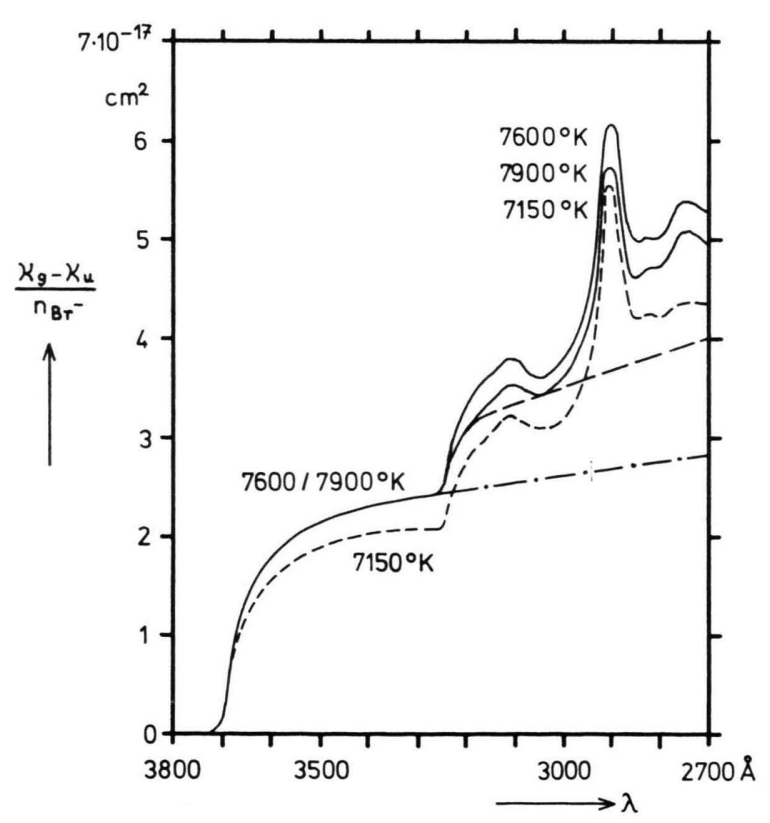

Abb. 6. Differenz zwischen Gesamtkontinuum und extrapoliertem Untergrund pro negatives Bromion oder mit Molekülbanden überlagerter Detachment-Querschnitt des negativen Bromions.

liegt um den Faktor 0,85 zu niedrig. Der Grund dafür ist, wie im Kapitel 2.8 gezeigt wird, in einer $\mathrm{Ab}$ weichung vom lokalen thermodynamischen Gleichgewicht zu suchen. Der Verlauf für $7150^{\circ} \mathrm{K}$ scheidet damit für alle weiteren Auswertungen aus.
Nach der zweiten langwelligen Kante ergibt sich jedoch auch für die beiden höchsten Temperaturen ein temperaturabhängiger Verlauf, der auf die Temperaturabhängigkeit der Molekülbanden zurückzuführen ist und der damit wiederum ein Unterfahren der Molekülbanden zur Abtrennung unmöglich macht. Um trotzdem eine vernünftige Abtrennung der Molekülbanden zu erhalten, wird der zwischen der ersten und zweiten langwelligen Kante ungestörte und temperaturunabhängige Verlauf des zum ${ }^{2} \mathrm{P}_{3 / 2}^{0}-{ }^{1} \mathrm{~S}_{0}$-Übergang gehörenden Detachment-Querschnittes $\left(\sigma_{\operatorname{det} 1}\right)$ durch eine analytische Funktion beschrieben und extrapoliert. Folgende empirische Funktion gibt den Verlauf des Detachment-Querschnittes $\sigma_{\text {det } 1}$ bis zur zweiten langwelligen Kante gut wieder:

$$
\begin{aligned}
\sigma_{\operatorname{det} 1}(\lambda)=17,5 & \cdot 10^{-18} \frac{3683}{\lambda} \\
\cdot & \sqrt{\operatorname{arctg}\left(23,5\left(\frac{1}{\lambda}-\frac{1}{3683}\right)\right)}\left[\mathrm{cm}^{2}\right],
\end{aligned}
$$

wobei $\lambda$ in $\AA$-Einheiten einzusetzen ist.

Die empirische Funktion für $\sigma_{\operatorname{det} 1}$ ist nach der 2. langwelligen Kante in Abb. 6 strichpunktiert eingezeichnet. Um daraus den gesamten DetachmentQuerschnitt zu ermitteln, wird dem extrapolierten $\sigma_{\operatorname{det} 1} \mathrm{ab}$ der 2. langwelligen Kante die empirische Funktion in halber Größe (entsprechend dem Verhältnis der statistischen Gewichte der Brom-Grundzustände) überlagert, und man erhält den in Abb. 6 gestrichelt eingezeichneten und unter den Molekülbanden liegenden und damit abgetrennten Verlauf für den gesamten Detachment-Querschnitt.

\subsection{Detachment- und Attachment-Querschnitte}

Der nach den vorstehenden Methoden vom Untergrund und den Molekülbanden abgetrennte Verlauf des Detachment-Querschnittes für negative Bromionen ist in Abb. 7 nochmals aufgezeichnet und kann mit den im Niederstrombogen gemessenen Detachment-Querschnitten von negativen Fluor- ${ }^{2}$ und Chlorionen ${ }^{3}$ verglichen werden (ausgezogene Kurven). Die Detachment-Querschnitte der negativen Fluor-, Chlor- und Bromionen werden unabhängig von der Elektronenaffinität der Atome mit wachsendem Ionenradius größer.

Die gemessenen Querschnitte (ausgezogen) lassen sich mit den Ergebnissen der Rechnungen von RoBINSON und GELTMAN ${ }^{6}$ (gestrichelt) vergleichen, bei welchen bereits die experimentell beobachteten 


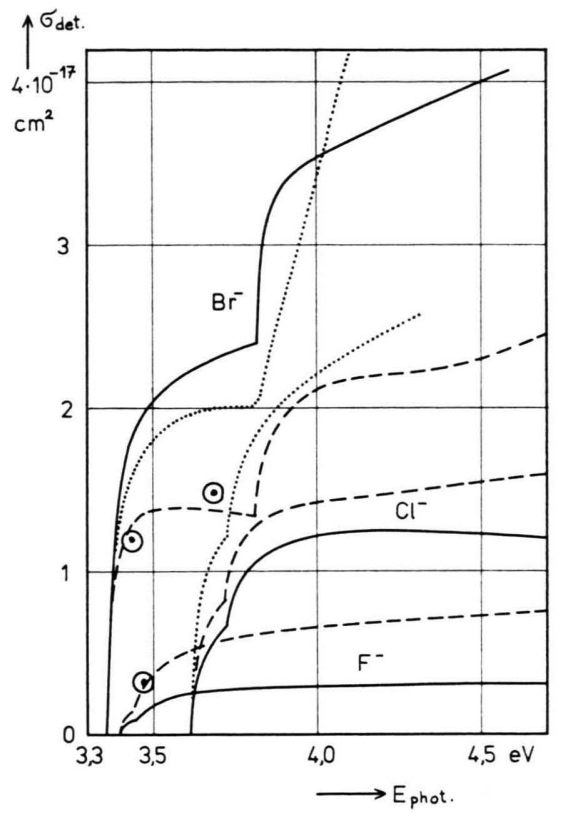

Abb. 7. Detachment-Querschnitte der negativen Ionen Fluor, Chlor, Brom: Messungen am Niederstrombogen - ausgezogen. Theorie von Robinson u. Geltman - gestrichelt. Messungen am Stoßwellenrohr von Rothe - gepunktet, und von Berry - Kreise.

Elektronenaffinitäten von BERRY ${ }^{17}$ verwendet wurden. Die Übereinstimmung der gemessenen Querschnitte mit der Theorie ist beim Chlor relativ gut, bei Brom und Fluor liegt die Theorie um einen Faktor 2 darunter bzw. darüber.

Die Ergebnisse der Messungen am Niederstromlichtbogen für Chlor und Brom lassen sich noch mit Messungen am Stoßwellenrohr von RoTHE ${ }^{4}$ (punktiert) und BERRY ${ }^{14}$ (Kreise) vergleichen. Für Brom ist die Übereinstimmung relativ gut. Der steile Anstieg der Bromquerschnitte von ROTHE nach der 2. langwelligen Kante dürfte auf die nicht abgetrennten Molekülkontinua bzw. Banden zurückzuführen sein.

Mit dem Prinzip des detaillierten Gleichgewichtes ${ }^{25}$ lassen sich die im Lichtbogen gemessenen Detachment-Querschnitte in den die Anlagerung der Elektronen an Atome beschreibenden AttachmentQuerschnitt $\sigma_{\text {att }}$ umrechnen.

$$
\sigma_{\mathrm{att}}=\left(\frac{E_{\mathrm{ph}}}{m c v_{\mathrm{el}}}\right)^{2} \frac{g^{-}}{g^{0}} \sigma_{\mathrm{det}} .
$$

Dabei bedeuten: $E_{\mathrm{ph}}=$ Energie des emittierten bzw. absorbierten Photons; $v_{\mathrm{el}}=$ Geschwindigkeit des

25 E. A. Milne, Phil. Mag. 47, 209 [1924]. freien Elektrons vor der Anlagerung bzw. nach der Ablösung; $g^{0}=$ Statistisches Gewicht des Atoms; $g^{-}=$Statistisches Gewicht des negativen Ions.

Führt man die Berechnung des Attachment-Querschnitts für die beiden zu den Übergängen ${ }^{2} \mathrm{P}_{3 / 2}^{0}{ }^{1} \mathrm{~S}_{0}$ und ${ }^{2} \mathrm{P}_{1 / 2}^{0}-{ }^{1} \mathrm{~S}_{0}$ gehörenden Detachment-Querschnitte getrennt durch, so erhält man bei beiden Rechnungen einen gleich großen Attachment-Querschnitt. Die beiden Grundzustände ${ }^{2} \mathrm{P}_{3 / 2}^{0}$ und ${ }^{2} \mathrm{P}_{1 / 2}^{0}$ der Halogen. atome besitzen also den gleichen Attachment-Querschnitt gegenüber freien Elektronen gleicher kinetischer Energie. Um den sich mit der Elektronenenergie stark ändernden Attachment-Querschnitt besser darstellen zu können, wurde in Abb. 8 eine doppeltlogarithmische Darstellung gewählt, bei der im be-

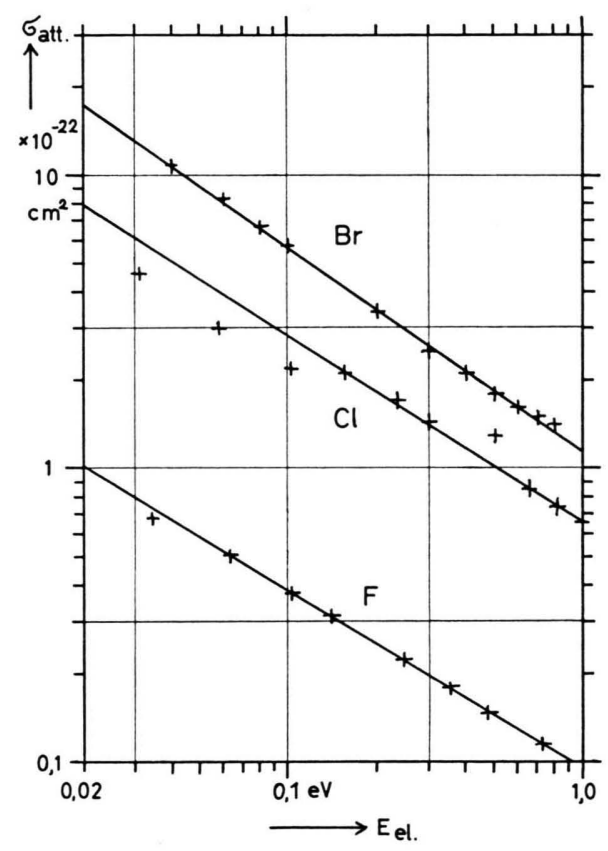

Abb. 8. Aus den Detachment-Querschnitten des Niederstromlichtbogens berechnete Attachment-Querschnitte für Fluor-, Chlor- und Bromatome.

trachteten Energiebereich der Attachment-Querschnitt auf einer Geraden liegt, deren Neigung für die drei gemessenen Halogene praktisch gleich ist. Im dargestellten Bereich der Elektronenenergien kann man aus den Steigungen der Attachment-Querschnitte entnehmen, daß sich die Attachment-Querschnitte mit $E_{\mathrm{el}}^{-0,7}$ oder mit $1 / v_{\mathrm{el}}^{1,4}$ ändern. In kantennahen $\mathrm{Ge}$ bieten, d. h. bei Elektronenenergien kleiner als $0,015 \mathrm{eV}$ ändern sich die Attachment-Querschnitte in Ubereinstimmung mit der Theorie (Wignersches 
Parabelgesetz ${ }^{26}$ ) mit $E_{\mathrm{el}}^{-0,5}$ bzw. umgekehrt proportional zur Elektronengeschwindigkeit. Der in Abb. 8 gezeigte Verlauf geht also zu kleineren Elektronenenergien hin in eine flacher geneigte Gerade über.

\subsection{Bremskontinuum und mittlerer totaler elastischer Streuquerschnitt}

Der im Bereich von $5300 \AA$ bis zur Grenze des Affinitätskontinuums quantitativ gemessene Absorptionskoeffizient des Untergrundes sowie die vorgenommene lineare Extrapolation zu kürzeren Wellenlängen hin ist in Abb. 9 ausgezogen bzw. gestrichelt eingezeichnet. Die Deutung des Untergrundes mit der Kontinuumstheorie der Elektronen und positiven Ionen (frei-gebunden und frei-frei-Kontinuum) nach KRAMERS $^{7}$ und UNSÖLD ${ }^{8}$ ist nicht möglich, da der nach dieser Theorie für die gemessenen Plasmatemperaturen berechnete Absorptionskoeffizient ein bis zwei Zehnerpotenzen unter den gemessenen Werten bleibt. Die bei den Chlormessungen ${ }^{3}$ bereits geäußerte Hypothese, daß der gemessene Untergrund weitgehend aus dem Bremskontinuum der Elektronen im Feld der Atome besteht (frei-frei-minusKontinuum), läßt sich hier beim Bromuntergrund mit Hilfe der Theorie von Kas'yanov und StaroSTIN ${ }^{11}$ weitgehend bestätigen.

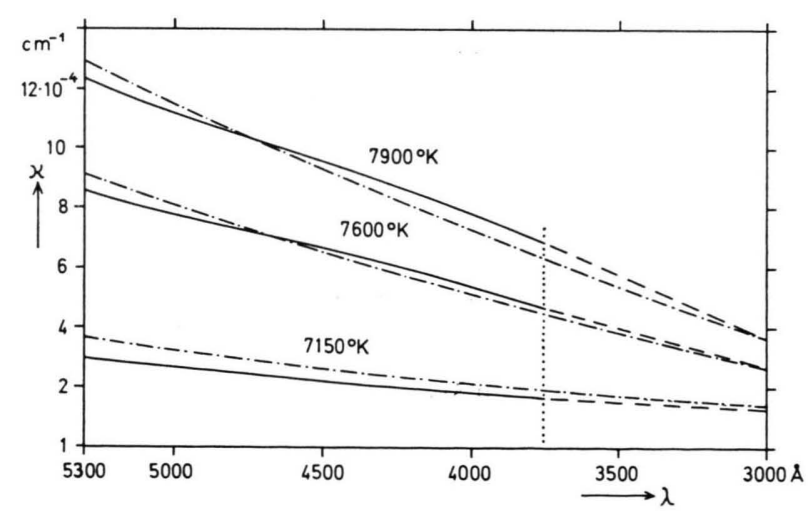

Abb. 9. Gemessener Untergrund (augezogen), Extrapolation (gestrichelt) und mit der Theorie von Kas'yanov und Starostin berechneter Untergrund (strichpunktiert).

Da die Bremsung des Elektrons im Feld des Bromatoms geschieht, ist der Emissionskoeffizient der Bremsstrahlung sowohl der Elektronen- als auch der Atomkonzentration proportional. Für die spektralen Emissionskoeffizienten läßt sich schreiben:

$$
\varepsilon_{(\text {ff-minus })} \sim \frac{\mathrm{d} \sigma_{\mathrm{e}, \mathrm{Br}}}{\mathrm{d} v} n_{\mathrm{e}} n_{\mathrm{Br}} .
$$

KAS'YANOV und STAROSTIN ${ }^{11}$ haben nach vorbereitenden Arbeiten anderer Verfasser einen mit der Erfahrung gut übereinstimmenden Zusammenhang zwischen dem differentiellen inelastischen Streuquerschnitt $\mathrm{d} \sigma_{\mathrm{e}, \mathrm{a}} / \mathrm{d} \boldsymbol{v}$ und dem totalen elastischen Streuquerschnitt $\sigma_{\mathrm{e}, \mathrm{a}}^{\text {elast }}$ für Elektronen-Atom-Streuung angegeben:

$\frac{\mathrm{d} \sigma_{\mathrm{e}, \mathrm{a}}(E, v)}{\mathrm{d} v}=\frac{4 \alpha}{3 \pi} \frac{E}{m c^{2}}\left(1-\frac{h v}{E}\right)^{1 / 2}\left(2-\frac{h v}{E}\right) \frac{\sigma_{\mathrm{e}, \mathrm{a}}^{\text {elast. }}}{v}(E)$.

Dabei bedeutet: $v=$ Frequenz der beim inelastischen Stoß emittierten Strahlung; $E=m v_{1}^{2} / 2$, kinetische Energie des Elektrons vor dem Streuprozeß; $\alpha=$ Feinstrukturkonstante.

Betrachtet man $\sigma_{\mathrm{e}, \mathrm{a}}^{\text {elast. }}(E)$ im in Frage kommenden Energiebereich als eine wenig von der Energie abhängige Funktion, dann kann man sie durch einen passend gewählten Mittelwert $\left\langle\sigma_{\mathrm{e}, \mathrm{a}}^{\text {elast. }}\right\rangle$ ersetzen. Mit dieser Vereinfachung erhalten die Verfasser ${ }^{11}$ einen geschlossenen analytischen Ausdruck für den spektralen Emissions- und Absorptionskoeffizienten des frei-frei-minus-Kontinuums.

Wählt man den mittleren elastischen Streuquerschnitt für Brom so, daß man bei $\lambda=4700 \AA$ Übereinstimmung der theoretischen Werte mit den gemessenen Werten des Kontinuums erhält (Abb. 9), so ergibt sich ein Wert von

$$
\left\langle\sigma_{\Theta, \mathrm{Br}}^{\text {elast }}\right\rangle=1,65 \cdot 10^{-14} \mathrm{~cm}^{2} .
$$

Abb. 9 zeigt, daß mit diesem hohen Wert eine Übereinstimmung des theoretischen Verlaufs des Absorptionskoeffizienten der frei-frei-minus-Strahlung (strichpunktierte Kurven) mit den Meßwerten des Untergrundes für die Wellenlängen und Temperaturabhängigkeit erhalten wird. Die Abbildung zeigt weiter die Berechtigung der angewendeten linearen Extrapolation, da die Abweichung vom gerechneten Verlauf innerhalb der Fehlergrenze liegt $(<10 \%)$. Die Unsicherheit bei der Abtrennung von maximal 10\% wirkt sich im Absorptionskoeffizienten des Affinitätskontinuums nur noch mit $1-3 \%$ aus, da das Verhältnis der Kontinua zwischen 10 und 3,5 liegt. In Abb. 10 ist ein Vergleich zwischen dem Emissionskoeffizienten des berechneten frei-frei-minusKontinuums und dem berechneten aber vernachlässigten Kramers-Unsöld-Kontinuum in der üblichen logarithmischen Auftragung über der Frequenz dargestellt.

Der große mittlere elastische Streuquerschnitt von Brom, der zur Deutung des Untergrundes notwendig ist, läßt sich in der Literatur nicht bestätigen. 


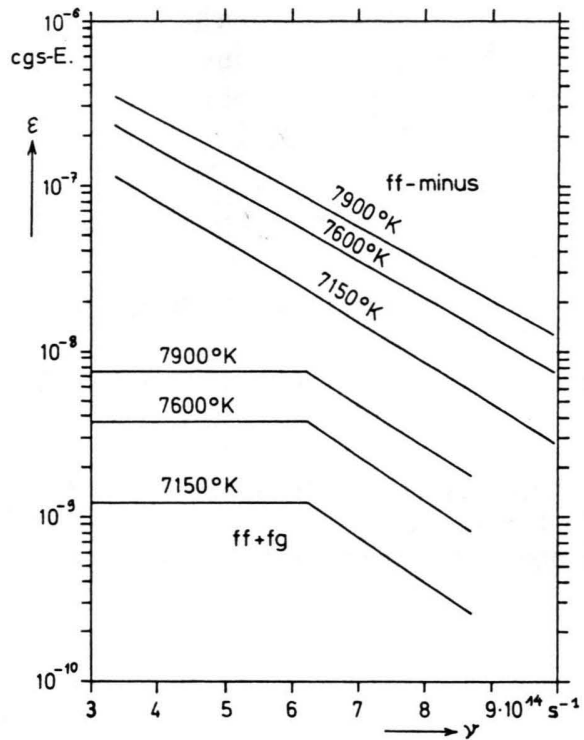

Abb. 10. Vergleich des nach Kas'yanov und Starostin berech. neten Untergrundes für frei-frei-minus-Strahlung mit der nach Kramers und Unsöld berechneten frei-frei- und frei-gebundenStrahlung.

Robinson und Geltman ${ }^{5}$ berechnen einen elastischen Streuquerschnitt für Brom bei ca. $8 \mathrm{eV}$ Elektronenenergie von

$$
\sigma_{\mathrm{e}, \mathrm{Br}}^{\text {elast. }}=2,7 \cdot 10^{-15} \mathrm{~cm}^{2} \text {. }
$$

Messungen des spektralen kontinuierlichen Untergrundes weit in den infraroten Spektralbereich hinein sollten hier die Diskrepanzen klären können.

\subsection{Thermisches Gleichgewicht}

Bei der Trennung des Affinitätskontinuums vom Untergrund erhielt man für die niedrigste Brombogentemperatur eine Abweichung des DetachmentQuerschnitts vom temperaturunabhängigen Wert (Abb. 6). Der Detachment-Querschnitt für $7150^{\circ} \mathrm{K}$ bzw. 7 Ampere Bogenstrom liegt um den Faktor 0,85 unter dem Wert für 7600 und $7900{ }^{\circ} \mathrm{K}$ bzw. 14,5 und 23,5 Ampere. Diese Abweichung erklärt sich mit einem unvollständigen lokalen thermischen Gleichgewicht, d. h. die Temperatur, die den Translationsbewegungen der schweren Teilchen (z. B. negativen Ionen) entspricht, liegt unter der spektroskopisch gemessenen Elektronentemperatur. Der zur niedrigsten Temperatur gehörende Detachment-

26 E. P. Wigner, Phys. Rev. 73, 1002 [1948].

27 K. Behringer, W. Kollmar u. J. Mentel, Z. Phys. 215, 127 [1968].
Querschnitt erreicht dann den temperaturunabhängigen Wert der höheren Temperaturen, wenn für die Teilchendichte der negativen Ionen ein Wert eingesetzt wird, wie er bei einer Gastemperatur von $6600{ }^{\circ} \mathrm{K}$ und nicht wie bei $7150^{\circ} \mathrm{K}$ im Teilchendichtediagramm auftritt.

MENTEL ${ }^{27}$ hat eine Formel angegeben, nach der sich die Abweichung der Gastemperatur von der Elektronentemperatur bei bekannter elektrischer Feldstärke im Lichtbogen und bei bekannten Wirkungsquerschnitten für alle Prozesse, die kinetische Energie von den Elektronen auf die schweren Teilchen übertragen, gaskinetisch exakt berechnen läßt.

Beschränken wir uns auf die Energieübertragung durch elastische Elektron-Atom-Stöße und ElektronIonen-Stöße, so wird diese Formel größere Abweichungen liefern als tatsächlich vorliegen. Mit der gemessenen Charakteristik (Abb. 2), den spektroskopisch gemessenen Elektronentemperaturen und einem Wirkungsquerschnitt von Bromatomen für elastische Stöße mit thermischen Elektronen von $\sigma_{\mathrm{e}, \mathrm{Br}}=5 \cdot 10^{-16} \mathrm{~cm}^{2} 28$ (Ramsauer-Minimum!) sowie einem mittleren Coulombquerschnitt von positiven Bromionen gegen Elektronen für Temperaturen zwischen 7000 und $8000^{\circ} \mathrm{K}$ von $\sigma_{e, \mathrm{Br}^{+}}=2,7 \cdot 10^{-13}$ $\mathrm{cm}^{2} 28$ erhält man die in Abb. 11 gezeigte Abweichung der Gastemperatur von der Elektronentemperatur im verwendeten Stromstärkebereich. Nach den Rechnungen erhält man selbst für die Stromstärke von 23,5 Ampere eine Abweichung vom LTG. Wie eingangs gezeigt, liegen die Verhältnisse beim

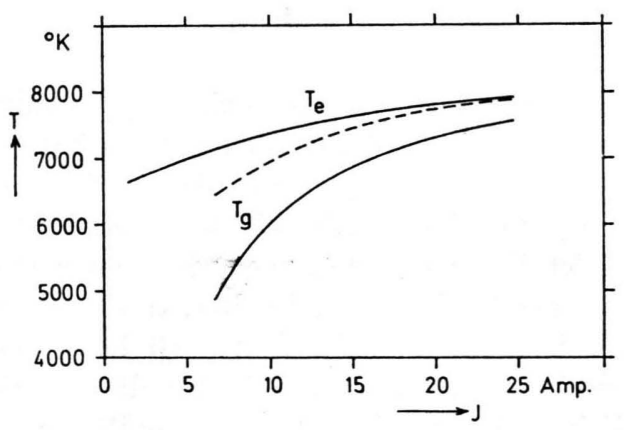

Abb. 11. Berechnete Abweichung vom lokalen thermischen Gleichgewicht (ausgezogen: Gas- und Elektronentemperatur) als Funktion der Bromlichtbogen-Stromstärke $(8 \mathrm{~mm} \phi)$ und wahrscheinlicher Verlauf der Gastemperatur beim Experiment (gestrichelt).

28 H. Maecker, Th. Peters u. H. Schenk, Z. Phys. 140, 119 [1955]. 
Experiment günstiger. Die gestrichelte Kurve in Abb. 11 gibt etwa den wirklichen Verlauf der Gastemperatur wieder. Das Experiment und selbst die ungünstige Abschätzung zeigen, daß die Auswertung der Detachment-Querschnitte aus den spektroskopischen Messungen zumindest für die größte gemessene Achsentemperatur mit keinem wesentlichen Fehler durch eine Abweichung vom LTG behaftet ist.

\section{Fehlerbetrachtung}

Für die Temperaturmessung ergibt sich eine relative Unsicherheit von $\pm 1 \%$ bzw. $\pm 80^{\circ} \mathrm{K}$ und damit für den Gesamtabsorptionskoeffizienten ein Fehler von $\pm 15 \%$. Mit den Unsicherheiten bei der Trennung des Affinitätskontinuums und des Teilchendichtediagramms ergibt sich ein Fehler für die Wirkungsquerschnitte von $\pm 22 \%$.

Die langwelligen Grenzen des Affinitätskontinuums bei 3682 und $3240 \AA$ konnten mit einer Unsicherheit von $\pm 2 \AA$ gemessen werden. In Photonenenergien umgerechnet erhält man $3,366 \pm 0,003 \mathrm{eV}$ sowie $3,825 \pm 0,004 \mathrm{eV}$. Der Fehler bei der Elektronenaffinität von Brom $\mathrm{EA}_{\mathrm{Br}}=3,366 \mathrm{eV}$ beträgt somit nur $0,1 \%$.

Die Verfasser danken Herrn Professor H. MAeckeR für die Förderung dieser Arbeit sowie der Deutschen Forschungsgemeinschaft für die bereitgestellten Mittel.

\title{
Frequenzmodulation und Kompression ultrakurzer Lichtimpulse
}

\author{
A. Laubereau und D. von Der Linde \\ Physik-Department der Technischen Universität München, Germany \\ (Z. Naturforsch. 25 a, 1626-1642 [1970]; eingegangen am 10. August 1970)
}

\begin{abstract}
Expressions are given for the phase modulation by the optical Kerr effect and the resulting frequency broadening of an optical pulse with elliptical polarization. The compression method is discussed for a quantitative comparison with experimental investigations. Starting with single pulses of $20 \mathrm{ps}$ duration and $0.4 \mathrm{~cm}^{-1}$ frequency width, generated by a mode-locked laser and an optical switching device, the phase modulation in an external cell containing $\mathrm{CS}_{2}$ produced a frequency broadening of $5 \mathrm{~cm}^{-1}$ typically. A compression factor of 5 is achieved in a system consisting of one optical grating and a reflection prism. Satisfactory agreement between theory and experiment is obtained for the frequency modulation $\Delta \omega$ and the compression length $l$. Our data suggest that the time dependence of our input pulses is symmetric with wings of higher power than those of a Gaussian pulse.
\end{abstract}

\section{Einführung}

Der momentane Zustand eines physikalischen Systems läßt sich mit Signalen untersuchen, deren Dauer kurz ist im Vergleich zu den Zeitkonstanten, die den physikalischen Prozeß charakterisieren. Daher findet die Erzeugung kurzer elektromagnetischer Impulse großes Interesse. Im Bereich der optischen Signale hat innerhalb der letzten 10 Jahre der Laser ${ }^{1,2}$ einen großen Fortschritt für die experimentelle Zeitauflösung gebracht. Über den Riesenimpuls-

Reprints request to Dr. A. Laubereau, Physik-Department der Technischen Universität München, D-8000 München 2, Arcisstraße 21.

1 A. L. Schawlow u. C. H. Townes, Phys. Rev. 112, 1940 [1958].

2 T. H. Maiman, Nature London 187, 493 [1960].

3 F. J. McClung u. R. W. Hell warth, J. Appl. Phys. 33, 828 [1962]. laser ${ }^{3}$ mit Pulsdauern von $10^{-8} \mathrm{~s}$ hat die Entwicklung zum phasengekoppelten Laser ${ }^{4,5}$ mit Signaldauern von der Größenordnung $10^{-12} \mathrm{~s}$ geführt. Nach Möglichkeiten zur Erzeugung noch kürzerer Lichtimpulse wird gesucht.

Die vorliegende Arbeit diskutiert die Methode der Impulskompression, die eine wesentliche Verkürzung optischer Signale gestattet. Die Methode wurde erstmals in der Radartechnik angewandt, wobei eine Pulsverkürzung bis zu einem Faktor 60 erreicht wurde ${ }^{6}$. Die Kompression von

4 M. DiDomenico, J. Appl. Phys. 33, 2870 [1964]. - L. E. Hargrove, R. L. Fork u. M. Pollack, Appl. Phys. Letters 5, 4 [1964].

5 A. J. DeMaria u. D. A. Stetser, Appl. Phys. Letters 7, 71 [1965]. - A. J. DeMaria, C. M. FerRar u. G. E. Danielson, Appl. Phys. Letters 8, 22 [1966].

6 J. R. Klauder, A. C. Price, S. Darlington u. W. J. AlBersheim, Bell Syst. Techn. J. 39, 745 [1960]. 